\title{
Introducing ROC Curves as Error Measure Functions: A New Approach to Train ANN-Based Biomedical Data Classifiers
}

\author{
Raúl Ramos-Pollán ${ }^{1}$, Miguel Ángel Guevara-López ${ }^{2}$, and Eugénio Oliveira ${ }^{3}$ \\ ${ }^{1}$ CETA-CIEMAT Centro Extremeño de Tecnologías Avanzadas, \\ Calle Sola 1, 10200 Trujillo, Spain, \\ raul.ramos@ciemat.es \\ 2 INEGI Instituto de Engenharia, Mecanica e Gestão Industrial, Universidade do Porto, \\ Campus da FEUP, Rua Roberto Frias 400, 4200-465 Porto, Portugal \\ mguevaral@inegi.up.pt \\ ${ }^{3}$ LIACC-DEI-Faculdade de Engenharia, Universidade do Porto, Rua Roberto Frias s/n, \\ 4200-465 Porto, Portugal \\ eco@fe.up.pt
}

\begin{abstract}
This paper explores the usage of the area (Az) under the Receiver Operating Characteristic (ROC) curve as error measure to guide the training process to build machine learning ANN-based classifiers for biomedical data analysis. Error measures (like root mean square error, RMS) are used to guide training algorithms measuring how far solutions are from the ideal classification, whereas it is well known that optimal classification rates do not necessarily yield to optimal Az's. Our hypothesis is that Az error measures can guide existing training algorithms to obtain better Az's than other error measures. This was tested after training 280 different configurations of ANN-based classifiers, with simulated annealing, using five biomedical binary datasets from the UCI machine learning repository with different test/train data splits. Each ANN configuration was trained both using the $\mathrm{Az}$ and RMS based error measures. In average $\mathrm{Az}$ was improved in $7.98 \%$ in testing data (9.32\% for training data) when using $70 \%$ of the datasets elements for training. Further analysis reveals interesting patterns (Az improvement is greater when $\mathrm{Az}$ are lower). These results encourage us to further explore the usage of Az based error measures in training methods for classifiers in a more generalized manner.
\end{abstract}

Keywords: ROC Curves, Artificial Neural Networks, Machine learning Classifiers, Biomedical Data.

\section{Introduction}

After preliminary data preparation, pattern recognition systems consist of two major stages: (1) feature extraction and selection and (2) classification. This means that a set of features is extracted from the pattern to be recognized and then classified into one of the possible classes. To achieve high recognition accuracy, the feature extractor is 
required to discover salient characteristics suited for classification and the classifier is required to set class boundaries accurately in the feature space. Progress made in sensor technology and data management allows researchers to gather datasets of ever increasing sizes [1].

The integration of biomedical information has become an essential task for health care, biology and biotechnology professionals and researchers. Integration is therefore much more than a plain collection of digital biomedical data. Homogenization of data description and storage, followed by normalization across the various experimental conditions would be a prerequisite to enable procedures of knowledge extraction [2].

The area under a ROC curve (or $A z$ [3]) is a decisive factor used in many applications to measure classifier quality (performance). However, it is known that optimal classification rates do not necessarily yield to optimal Az's [4] and a few attempts have tried to use $\mathrm{Az}$ in optimization problems [5-6]. This paper explores the usage of the ROC Az as error measure to guide the training process to build machine learning ANN-based classifiers for biomedical data analysis. Our hypothesis is that by doing this, we will obtain better Az's than those obtained through other error measures.

This paper is structured as follows. Section 2 establishes the theoretical background of this work. Section 3 describes the technological framework used to experimentally validate our hypothesis on a Grid infrastructure. Section 4 describes the experiments performed and Section 5 discusses the results obtained. Section 6 draws some conclusions and outlines future work.

\section{ROC Az Based Error Measures}

\subsection{ANNs Trained with Simulated Annealing}

Simulated annealing was first proposed in [9] and it is inspired by the physical process of annealing to find good values of functions depending on many parameters. In short, the algorithm includes a parameter, simulating temperature starting at a given value, which is lowered gradually at known steps. For each temperature, the function parameters are randomized and the range of possible values that they can take is proportional to the temperature, so that at lower temperatures that range is smaller. At each temperature step the process is repeated a predetermined number of times and the set of parameters giving the best function value are retained and passed on to the following cycle iteration.

We use the simulated annealing approach to train ANNs as described and implemented in [7] which the weights of an ANN population are randomized iteratively and, at each step, the ANN with the minimum root mean square (RMS) error is retained. Notice that, in this case, the error used by the algorithms is the RMS of the whole training set, as opposed to other algorithms such as backpropagation, where it is the individual RMS error of each element of the training set with respect to the output neurons the one that is used.

More formally, for binary classifiers, we use the following definitions: 
Table 1. Definitions

\begin{tabular}{cl}
\hline $\mathcal{X} \subset \mathbb{R}^{p}$ & Domain of input vectors (with $p$ features) \\
\hline $\mathcal{Y}=\{-1,1\}$ & The two classes into which input vectors are classified \\
\hline$\left.S=\left\{\left(x_{i}, y_{i}\right) x_{i}, y_{1}\right), \ldots,\left(x_{n}, y_{n}\right)\right\}$ & Training set (for supervised training) \\
\hline$|S|=n$ & Size of training set \\
\hline $\mathcal{F}=\{f: \mathcal{X} \rightarrow \mathcal{Y}\}$ & Set of functions representing binary classifiers \\
\hline$h \in \mathcal{F}$ & A binary classifier \\
\hline$h\left(x_{i}\right) \in \mathcal{Y}$ & $\begin{array}{l}\text { Output of binary classifier } h \text { when applied to input vector } x_{i}, \\
h\left(x_{i}\right) \text { typically applies some threshold notion to } h_{\text {score }}\left(x_{i}\right) \text { to } \\
\text { obtain the final class assigned to } x_{i}\end{array}$ \\
\hline$h_{s c o r e}\left(x_{i}\right) \in \mathbb{R}$ & Score assigned by binary classifier $h$ to input vector $x_{i}$, \\
\hline $\mathcal{E}(S, h)$ & $\begin{array}{l}\text { A global error measure of classifier } h \text { when applied to training } \\
\text { set } S\end{array}$ \\
\hline$e\left(x_{i}, h\right)$ & $\begin{array}{l}\text { An individual error measure of classifier } h \text { when applied to } \\
\text { input vector } x_{i}\end{array}$ \\
\hline$A z(S, h)$ & $\begin{array}{l}\text { Area under the ROC curve of training set } S \text { when classified } \\
\text { with classifier } h\end{array}$ \\
\hline
\end{tabular}

In particular, an RMS error measure is typically defined as follows:

$$
\mathcal{E}_{R M S}(S, h)=\frac{\sum e_{R M S}\left(x_{i}, h\right)}{|S|}
$$

where $e_{R M S}\left(x_{i}, h\right)$ represents some distance measure between $h_{\text {score }}\left(x_{i}\right)$ and $y_{i}$, possibly using the output values of the output neurons in case of ANN based classifiers. Algorithms such as backpropagation in ANN based classifiers use the individual values $e_{R M S}\left(x_{i}, h\right)$ iterating through each element of the training set to incrementally correct the ANN weights, whereas simulated annealing uses only the global $\mathcal{E}_{R M S}(S, h)$ value to select the best classifier at each cycle of each cooling step.

\subsection{ROC Az Error with Simulated Annealing}

We now use ROC Az to define a global error measure as follows:

$$
\mathcal{E}_{R O C}(S, h)=1-A z(S, h)
$$

and use this definition, instead of (1), as error measure in the simulated annealing based ANN training process described above. $\mathcal{E}_{R O C}$ is implemented in the ffsaroc engine included in Biomedtk (see Section 3) whereas $\mathcal{E}_{R M S}$ is used in the ffs $a$ engine.

Note that when using RMS there is a direct relation between the individual error measures of the elements of the training set $\left(e_{R M S}\right)$ and the global error $\left(\mathcal{E}_{R M S}\right)$ which is given by equation (1) and this is why it can be used by backpropagation-like 
training algorithms, whereas there is no such direct relation in $\mathcal{E}_{R O C}$ since $A z(S, h)$ is a global measure of a classified set. It is the fact that simulated annealing does not use individual error measures for each element of the training set that allows us to replace $\mathcal{E}_{R M S}$ by $\mathcal{E}_{R O C}$ in a straight forward manner.

\section{The Biomedtk Framework}

The Biomedical Data Analysis Toolkit (Biomedtk) is a Java software tool developed by the authors that exploits existing libraries for data analysis with methods and metrics commonly used in the biomedical field. In addition, it provides the means to massively search, explore and combine different configurations of data classifiers provided by the underlying libraries to build robust data analysis tools. With this, it is possible manipulate datasets, train Artificial Neural Networks (ANN) based binary and multiclass classifiers with many different configurations, search for best ensemble classifiers, generate different types of ROC curve analysis, etc.

An ANN-based configuration specifies a certain network structure (number of layers and neurons per layer), a training algorithm to use (such as backpropagation or simulated annealing) and algorithm dependant train parameters (such as learning rate, start/end temperatures, etc.). Biomedtk allows defining explorations of ANN configurations (see Section 4) and sending them for massive training to a Grid computing infrastructure. Currently, biomedtk supports training engines from the Encog [7] and Weka [8] toolkits, as listed in table 2.

Table 2. Biomedtk supported training engines

\begin{tabular}{ll}
\hline Engine name & Description \\
\hline ffbp & Feedforward with backpropagation-based training. \\
ffga & Feedforward with genetic algorithm-based training. \\
ffsa & Feedforward with simulated annealing-based training. \\
ffsaroc & ffsa with WEKA ROC based error evaluation. \\
Rb & Radial basis. \\
som & Self-organizing feature map. \\
\hline
\end{tabular}

It also includes the ffsaroc which is a modification of the ffs $a$ engine including the ROC Az based error evaluation described in Section 3. Biomedtk uses the MannWhitney statistic to calculate ROC Az as implemented in Weka[8].

\section{Experimental Setup}

A set of experiments was set up in order to test whether the error measure proposed in Section 2 effectively improves the Az of the trained ANN classifiers. The tests were carried out using the binary biomedical UCI datasets [10] listed in table 3. 
Table 4. FFSAROC improvement of FFSA per training set

\begin{tabular}{lrr|rr|rr}
\hline & \multicolumn{2}{c|}{ test Az increase } & train Az increase & \multicolumn{2}{|c}{ avg test Az } \\
Dataset & $a v g$ & std dev & avg & std dev & ffsaroc & ffsa \\
\hline haber-30 & $7.92 \%$ & 5.58 & $6.90 \%$ & 1.53 & 0.691 & 0.642 \\
haber-50 & $2.33 \%$ & 3.94 & $8.25 \%$ & 2.13 & 0.729 & 0.713 \\
liver-30 & $3.91 \%$ & 3.81 & $4.50 \%$ & 1.94 & 0.698 & 0.672 \\
liver-50 & $0.98 \%$ & 2.46 & $7.60 \%$ & 2.99 & 0.725 & 0.718 \\
mmass-30 & $3.24 \%$ & 5.43 & $6.83 \%$ & 3.27 & 0.807 & 0.782 \\
mmass-50 & $-0.59 \%$ & 4.94 & $7.46 \%$ & 2.78 & 0.786 & 0.790 \\
pimadiab-30 & $5.44 \%$ & 4.42 & $7.91 \%$ & 3.88 & 0.704 & 0.669 \\
pimadiab-50 & $1.45 \%$ & 6.69 & $11.83 \%$ & 5.35 & 0.691 & 0.684 \\
spectf-30 & $19.38 \%$ & 20.57 & $20.45 \%$ & 8.43 & 0.830 & 0.712 \\
spectf-50 & $8.34 \%$ & 17.35 & $25.85 \%$ & 16.40 & 0.757 & 0.711 \\
\hline total 30\% test sets & $7.98 \%$ & 11.77 & $9.32 \%$ & 7.27 & 0.746 & 0.695 \\
total 50\% test sets & $2.50 \%$ & 9.37 & $12.20 \%$ & 10.62 & 0.738 & 0.723 \\
\hline total all datasets & $\mathbf{5 . 2 4 \%}$ & $\mathbf{1 0 . 9 8}$ & $\mathbf{1 0 . 7 6 \%}$ & $\mathbf{9 . 2 1}$ & $\mathbf{0 . 7 4 2}$ & $\mathbf{0 . 7 0 9}$ \\
\hline
\end{tabular}

Table 5 shows the same classifier pairs grouped by ffsa testA $z$ value ranges.

Table 5. FFSAROC improvement over FFSA per FFSA Test Az range

\begin{tabular}{lc|rr|rr}
\hline $\begin{array}{l}\text { FFSA } \\
\text { Test Az } \\
\text { range }\end{array}$ & $\begin{array}{c}\text { Number of } \\
\text { Classifier } \\
\text { Pairs }\end{array}$ & \multicolumn{2}{|c|}{ test Az increase } & \multicolumn{2}{|c}{ train Az increase } \\
\hline $0.00,0.60]$ & 8 & $51.16 \%$ & std dev & $a v g$ & std dev \\
$(0.60,0.65]$ & 30 & $12.94 \%$ & 7.31 & $43.81 \%$ & 20.36 \\
$(0.65,0.70]$ & 82 & $5.17 \%$ & 6.77 & $9.73 \%$ & 6.13 \\
$(0.70,0.75]$ & 81 & $2.26 \%$ & 5.39 & $9.39 \%$ & 7.01 \\
$(0.75,0.80]$ & 67 & $1.28 \%$ & 5.90 & $9.80 \%$ & 5.34 \\
$(0.85,1.00]$ & 12 & $-1.96 \%$ & 5.65 & $14.76 \%$ & 6.79 \\
\hline total all ranges & $\mathbf{2 8 0}$ & $\mathbf{5 . 2 4 \%}$ & $\mathbf{1 0 . 9 8}$ & $\mathbf{1 0 . 7 6 \%}$ & $\mathbf{9 . 2 1}$ \\
\hline
\end{tabular}

As it can be seen in the tables above there is in general a significant average improvement in $\mathrm{Az}$ both for train and test parts of the datasets. Note that the improvement percentages shown are the average of the compared classifier pairs (between ffsaroc and ffsa trained classifiers with the same network structure and training parameters), which is different from the average test $A z$ of all ffsaroc and ffsa classifiers in each training set shown in the last two columns of table 4.

Improvement is constantly better when splitting datasets in 30/70 test/train data than when splitting in 50/50. Even if the 30/70 datasets have better average testAz than 50/50 (such as in the mmass, pimadiab and spectf datasets). Improvement is also constantly better in the train parts of the dataset than in the test parts and, in any case, its standard deviation is always high. This seems to indicate that the method presented in this paper may be hard for classifiers to generalize and does not behave homogeneously for all classifiers. From table 5, it can be clearly seen that for test data, improvement is better when the testA $z$ for the ffsa classified dataset is worse. Finally, the 
following plots show the ROC curves of the classifier pairs for which the best improvement was obtained (ffsaroc over ffsa) for the haber and spectf datasets both for the $30 / 70$ and $50 / 50$ data splits
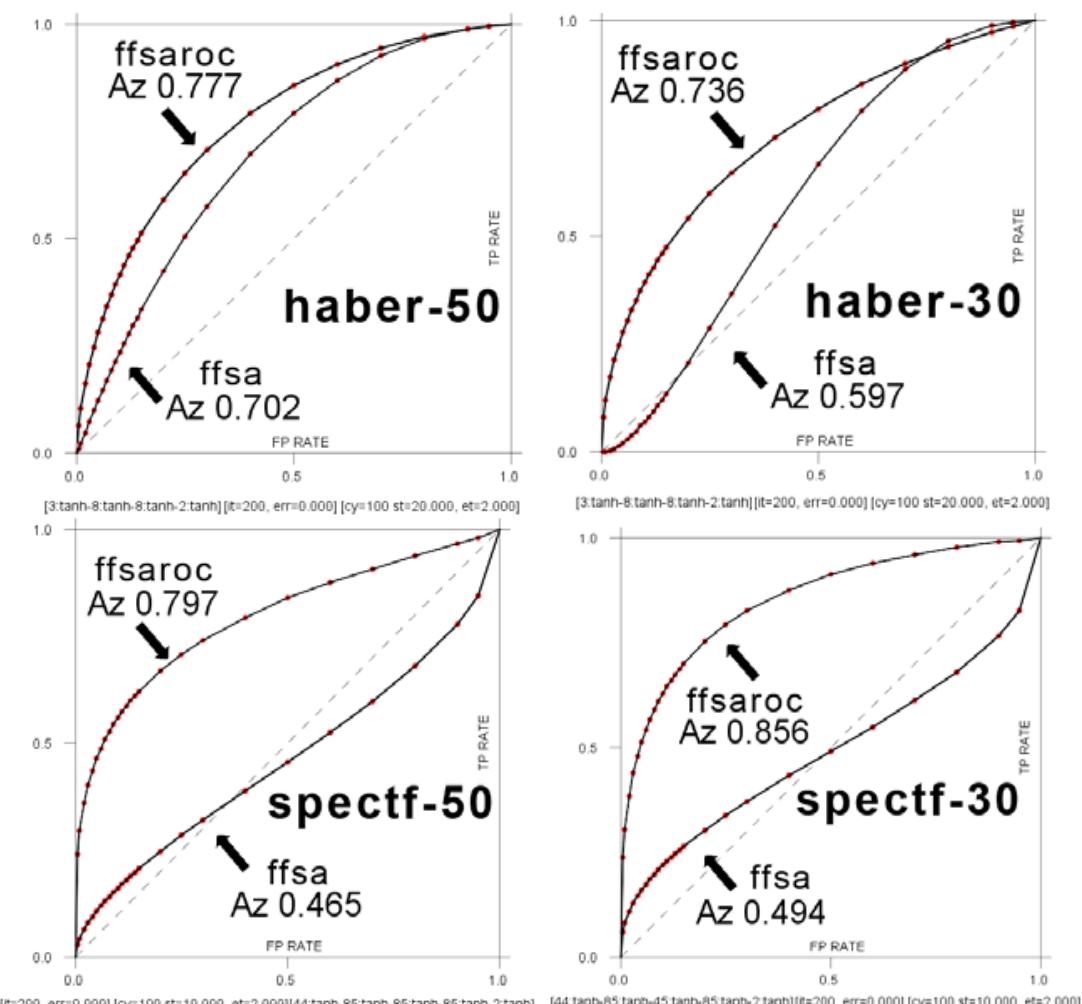

Fig. 1. Most improved classifiers for spectf and haber, 30\% and 50\% test data splits

To simplify visual comparison, these plots use the bi-normal distribution method as provided by JLABROC [12] which is also supported by Biomedtk. Also, each plot shows the ANN layers structure (neurons per layer and activation function) and the training parameters for the simulated annealing processes (start temperature, end temperature and number of cycles)

\section{Conclusions}

The experimental results obtained here confirm that the usage of a ROC Az based error function to guide a simulated annealing algorithm for training ANNs improves the ROC Az of the obtained classifiers with respect to an RMS error function. In addition, Biometk demonstrated to be a robust framework to massively explore large amounts of configurations of data classifiers exploiting computing power harnessed by Grid infrastructures. 
Future work is focused on (1) better understanding of the behavior of the proposed method to better explain deviations observed in the experiments and (2) on applying the method in a generalized manner to machine learning classifiers and validate them in real computer-aided detection/diagnosis systems.

\section{Acknowledgements}

Prof. Guevara acknowledges POPH - QREN-Typology 4.2 - Promotion of scientific employment funded by the ESF and MCTES, Portugal. Prof. Ramos-Pollán acknowledges the support of the European Regional Development Fund.

\section{References}

1. Kostka, P., Tkacz, E.J.: Feature extraction and selection algorithms in biomedical data classifiers based on time-frequency and principle component analysis. In: Proc. 11th Mediterranean Conference on Medical and Biomedical Engineering and Computing 2007, vol. 16, pp. 70-73. Springer, Heidelberg (2007)

2. Drakos, J., Karakantza, M., Zoumbos, N., Lakoumentas, J., Nikiforidis, G., Sakellaropoulos, G.: A perspective for biomedical data integration: Design of databases for flow cytometry. BMC Bioinformatics 9(1), 99 (2008)

3. Fawcett, T.: An introduction to ROC analysis. Pattern Recognition Letters 27(8), 861-874 (2006)

4. Castro, C.L., Braga, A.P.: Optimization of the Area under the ROC Curve. In: Proc. of 10th Brazilian Symposium on Neural Networks, SBRN 2008, pp. 141-146 (2008)

5. Cortes, C., Mohri, M.: AUC optimization vs. error rate minimization. In: Advances in Neural Information Processing Systems. MIT Press, Cambridge (2003)

6. Rakotomamonjy, A.: Optimizing Area under ROC Curve with SVMs. In: Proc. Workshop of ROC Analysis in Artificial Intelligence, pp. 71-80. ROCAI (2004)

7. Heaton, J.: Programming Neural Networks with Encog 2 in Java. Heaton Research, Inc. (2010)

8. Hall, M., Frank, E., Holmes, G., Pfahringer, B., Reutemann, P.: Witten, I.H.: The WEKA Data Mining Software: An Update. SIGKDD Explorations 11(1) (2009)

9. Kirkpatrick, S., Gelatt Jr., C.D., Vecchi, M.P.: Optimization by Simulated Annealing. Science 220(4598), 671-680 (1983)

10. Asuncion, A., Newman, D.J.: UCI Machine Learning Repository. University of California, School of Information and Computer Science, Irvine, CA (2007), http: / / www. ics.uci.edu/ mlearn/MLRepository.html

11. EGEE: The gLite middleware, vol. 2010 (2009)

12. John Eng, M.D.: ROC analysis: web-based calculator for ROC curves, vol. 2010. Johns Hopkins University, Baltimore (2006) 